\title{
Relative-Implant Anatomy in Panoramic and CBCT Radiographs: Knowledge and Attitude of Dental Students
}

ISSN: 2637-7764

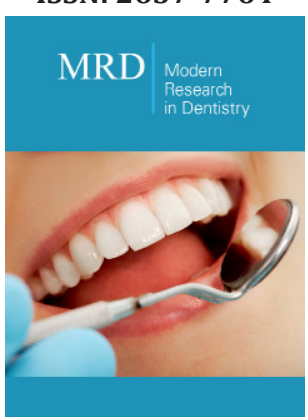

*Corresponding author: Rania Mostafa Moussa, Prince, Naif Ibn Abdulazia, Medinah, 42353, Saudi Arabia

Submission: 悳 January 26, 2021

Published: 留 February 04, 2021

Volume 6 - Issue 1

How to cite this article: Rania Mostafa Moussa, Sarah Yousef Al Hejaili, Lujain Atiq Alrehaili, Rawan Wadeea Tola, Manar Abdulrahman Alrashdi. RelativeImplant Anatomy in Panoramic and CBCT Radiographs: Knowledge and Attitude of Dental Students. Mod Res Dent. 6(1). MRD. 000628. 2021.

\section{DOI:_10.31031/MRD.2021.06.000628}

Copyright@ Rania Mostafa Moussa, This article is distributed under the terms of the Creative Commons Attribution 4.0 International License, which permits unrestricted use and redistribution provided that the original author and source are credited.

\author{
Rania Mostafa Moussa ${ }^{*}$, Sarah Yousef Al Hejaili ${ }^{2}$, Lujain Atiq Alrehaili², Rawan \\ Wadeea Tola ${ }^{2}$ and Manar Abdulrahman Alrashdi ${ }^{2}$ \\ ${ }^{1}$ Department of Substitutive Dental Sciences, College of Dentistry, Taibah University, Medinah, \\ Saudi Arabia \\ ${ }^{2}$ Dental graduate, College of Dentistry, Taibah University, Medinah, Saudi Arabia
}

\begin{abstract}
This study aimed to assess attitude and knowledge of sixth year dental students and interns towards panorama and CBCT, and to compare responses relative to level of study and gender. Web-based questionnaire comprising of four sections was used. First, participants provided demographic information. Second, ten close ended questions about panoramic radiography in personal practice and general information of CBCT. Third, identifying marked anatomical structures in digital panorama. Last, participants were asked to choose type of view and to name marked anatomical structure in CBCT images. Results showed that majority of participants used panorama in routine work, most did not think panorama was suitable for implant planning. 58.3\% students and 50\% interns did not use CBCT before. Both levels obtained knowledge on CBCT in faculty lessons, followed by internet. Future applications of CBCT reported highest in maxillofacial surgery for sixth year, and all fields of dentistry in interns. Most were willing to receive extended education in CBCT. High Correct identification of landmarks in panorama, while average correct identification of cross sections and marked landmarks in CBCT. It is concluded that the moderate students' knowledge of CBCT suggest that more training should be gained through continuing education.
\end{abstract}

Keywords: Panoramic radiographs; Cone beam computed tomography [CBCT]; Dental students; Dental implants

\section{Introduction}

Popularity of dental implants in rehabilitation of partial and completely edentulous patients, has increased over the last few years, in concurrence with increased demands of advanced technology and trained dentists. Comprehensive patient care and efficient implant planning relies on the use of appropriate dental radiographs and the capability to interpret radiographic findings. Successful implants placement requires recognition of adjacent structures thus permitting sufficient bone interface between implant fixtures and vital structures $[1,2]$.

Radiographic techniques available for the evaluation of dental implant patients are either plain 2-dimentional projections as dental panoramic radiographs and occlusal views, or a more advanced reformatted cross-sectional three dimensional [3D] imaging techniques as the computed tomography [CT], and cone bean computerized tomography [CBCT] [2].

Dental panoramic radiography is one of the most commonly used radiographic techniques. It is readily available, cost effective and with least radiation exposure. As far as implant placement is concerned, digital panoramic imaging provides data regarding crestal bone height relative to the inferior alveolar canal, gross anatomy of the jaws, any related pathologic findings, and adjacent anatomical landmarks are easily identified. The procedure is performed with convenience, ease and speed [3]. However, it renders a number of limitations on top of which are distortion, ghost shadows, superimposition and magnification [4].

The evolution of 3D imaging contributed to fulfilling the needs for high medical technology and enhanced the delivery of innovative treatment modalities. CT scanning was introduced at the beginning of the $20^{\text {th }}$ century. It offered the advantages of enhanced diagnosis and 
treatment planning of clinical procedures such as craniofacial reconstruction and placement of dental implants. However, high cost, limited access, and high radiation exposure, were the main drawbacks for underutilization of CT in dentistry [5].

CBCT was introduced as an alternative to medical CT and has been considered appropriate for dental applications. Major advantages offered by CBCT imaging for dental use were the rapid scan time, simple use, lesser radiation dose than conventional CT, and high image resolution. In addition, it occupies lesser space and can be easily mounted in dental offices [6,7].

With the increased utilization of dental implants retained and supported prosthesis, and the availability of CBCT in dental practice, it is necessary to determine the level of knowledge and attitude of dental students and freshly graduate dental interns towards these new technologies. Successful interpretation of diagnostic radiographs begins with the ability to identify normal anatomy of the maxillomandibular region.

The aim of this study was to assess the knowledge and attitude of sixth year dental students and interns towards digital panoramic and CBCT radiography assuming the null hypothesis that there is no difference.

\section{Material and Methods}

In a cross-section study, an anonymous web-based survey was used, that was designed to take approximately ten minutes to complete (Appendix 1). This study was conducted at the College of Dentistry, Taibah University, Medinah, Saudi Arabia. The research protocol was approved by Taibah University College of Dentistry Research Ethics Committee [TUCDREC].
The study addressed all male and female sixth year dental students [N=104], and dental interns [N=90]. Personal emails of students and interns as well as social media [WhatsApp messages] were used to reach the target sample and invited them to share voluntarily in the study.

The questionnaire was designed of four sections. In the first section, the participant consented to participate in the study and provided demographic information regarding their gender and year of education.

In the Second section, ten close ended questions were formulated to investigate general information and attitude of the participants regarding panoramic radiographs, their rate of use in personal dental practice and its suitability for implant planning from their point of view. As well as general information regarding CBCT, previous use in personal practice, and their sources of information on CBCT. Finally, personal opinions about the suitability of using CBCT in different dental practices, and their willingness to receive extended information and practice on CBCT imaging.

The third section was concerned with identifying implantrelated anatomical structures in digital panoramic radiographs. Four orthopatogramic images of partially edentulous patients were retrieved from the records of oral and maxillofacial radiology department. Patients' records were anonymous with guarantee of data confidentiality. Radiographic regions of interest were marked on the panoramic images (Figure 1a) and was accompanied by an unaltered copy to facilitate identification (Figure 1b). The responders were asked to report the name of the marked structure in the space provided, there were no multiple choices.

\section{Figure 1:}

a. Example of panoramic radiographic image showing region of interest marked by an arrow,

b. Un-altered copy of the same image. 
The last section of the survey included four CBCT images of partially edentulous patients that were retrieved from the records of oral and maxillofacial radiology department. Patients records were anonymous with guarantee of data confidentiality. In this section, the participants were asked to choose the type of view from four multiple choices [axial, sagittal, coronal, or panoramic], and to name the marked anatomical structure for each image (Figure $2 \mathrm{a}$ $\& 2 b)$.

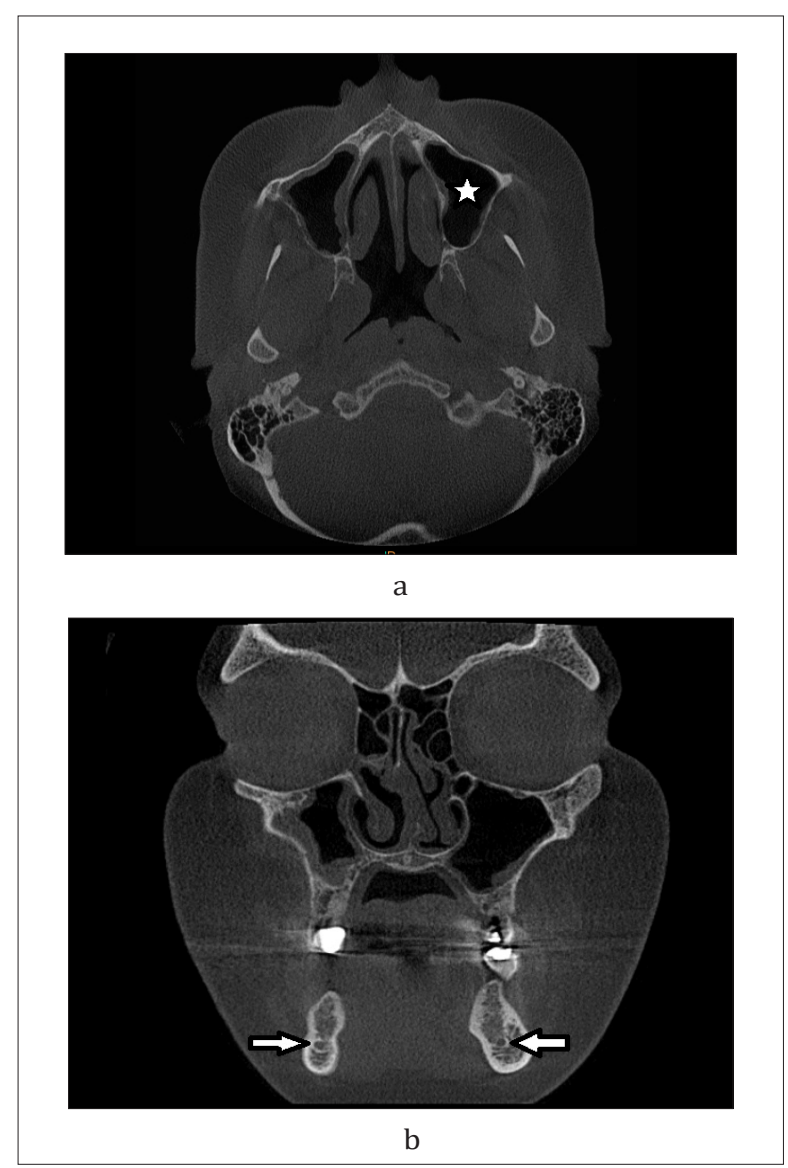

Figure 2: Examples of $\mathrm{CBCT}$ radiographic images showing regions of interest marked and the question was to choose the type of view and to identify the anatomical structure marked.

a. axial view, maxillary sinus,

b. coronal view, mandibualr canal.

All responses for the sections three and four were reported as correct or incorrect, and frequency of correct responses was reported.

The collected data was analyzed by Statistical Package for Social Sciences Version 16.0 [IBM SPSS v. 16.0]. The data analysis was performed according to descriptive statistics and presented as frequencies [n] and percentages [\%]. Students results were analyzed by Chi-square test to compare responses depending on level of education and gender. Level of significance was set at $\mathrm{P}<0.05$.

\section{Result}

This study addressed all male and female sixth year students and dental interns, out of 195 targeted students, 72 responses were received [response rate $36.9 \%$ ]. The distribution of students according to gender and level of education is shown in (Figure 3). There was significant increase in female responses relative to males in both levels.

Majority of the participants $95.8 \%$ [sixth year students $n=46$, interns $n=23$ ] knew that panorama means wide view. In response to the use of panoramic radiography in their routine examination of patients, $89.6 \%[n=43]$ of the sixth-year students and $87.5 \%[n=21]$ interns reported [Yes]. No significant difference between males and females $[\mathrm{P}=0.439]$ or between 6 th year and interns [ $\mathrm{P}=0.073]$. Only two female interns denied the use of panoramic radiography in their routine work.

Majority of participants did not agree that panoramic radiography is ideal for implant planning. Detailed responses are shown in (Figure 4). 


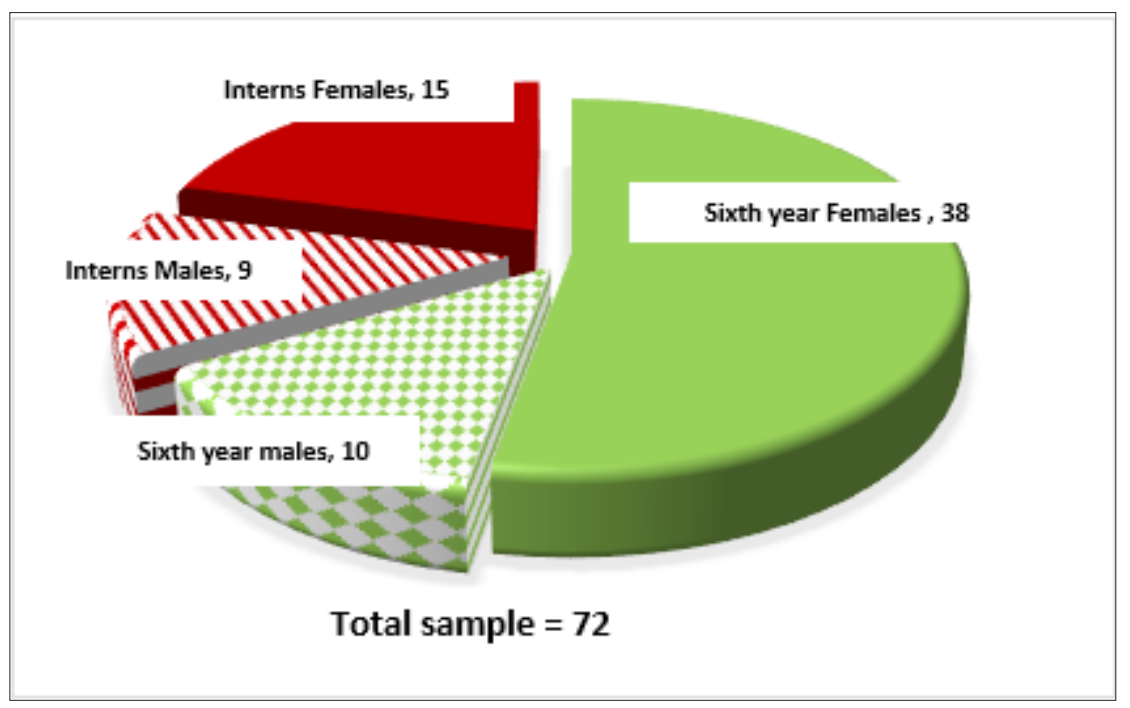

Figure 3: Pie chart distribution of students according to gender and level of education in the responding sample.

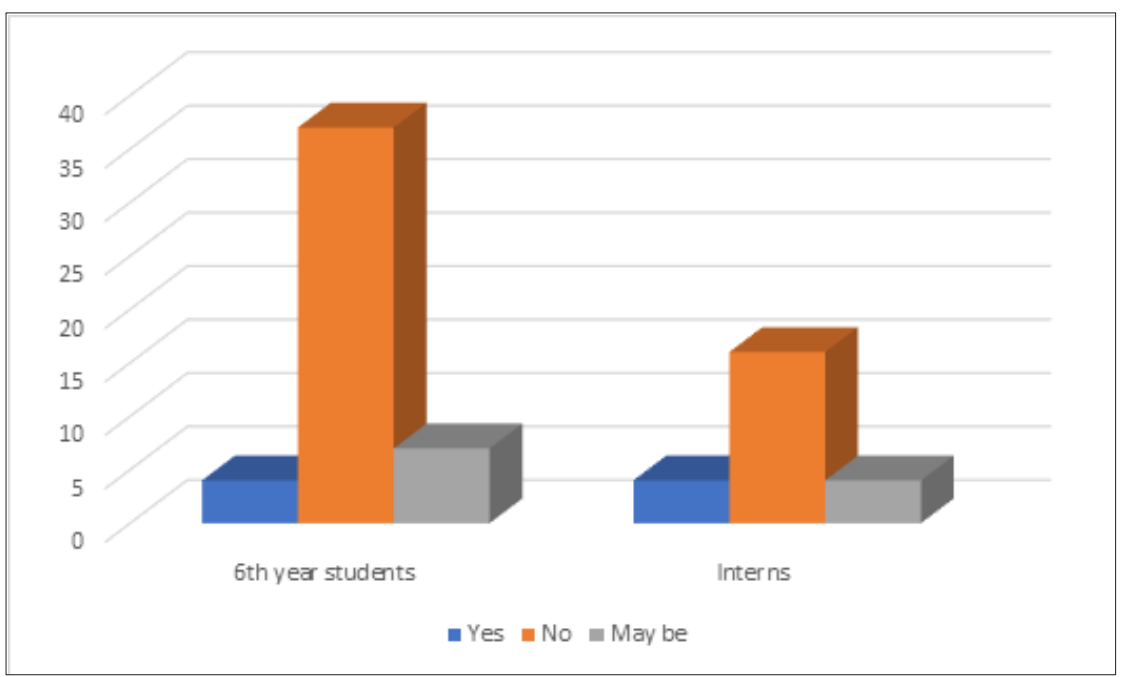

Figure 4: Bar chart students' response to question: is panoramic radiography ideal in implant planning?

Table 1 shows responses to questions [4-6] of the second part of the questionnaire, concerned with students' attitude towards CBCT; Have you heard of CBCT used specifically for implant planning? what does CBCT abbreviation stands for? Have you used CBCT in your work before with any of your patients? $95.75 \%$ of sixth year students and $87.5 \%$ of interns have heard of CBCT used specifically in implant planning. There were no significant differences between males and females or between levels of education.

Table 1: Responses to questions [4-6] concerned with students' attitude towards CBCT.

\begin{tabular}{|c|c|c|c|c|c|c|}
\hline \multirow{2}{*}{ Question } & \multirow{2}{*}{ Response } & \multicolumn{2}{|c|}{ Sixth year } & \multicolumn{2}{|c|}{ Interns } & \multicolumn{2}{|c|}{ Total } \\
\cline { 2 - 6 } & & Males & Females & Males & Females & N, \% \\
\hline \multirow{2}{*}{$\begin{array}{c}\text { Q4. Have you heard of CBCT used specifically for } \\
\text { Implant planning? }\end{array}$} & Yes & $9[90 \%]$ & $36[94.7 \%]$ & $8[88.89 \%]$ & $13[86.67 \%]$ & $66[91.6 \%]$ \\
\cline { 2 - 7 } & No & $1[10 \%]$ & $2[5.3 \%]$ & $1[11.11 \%]$ & $2[13.3 \%]$ & $6[8.3 \%]$ \\
\hline \multirow{2}{*}{\begin{tabular}{c} 
Q5. What does CBCT abbreviation stands for? \\
\cline { 2 - 6 }
\end{tabular}} & Yes & $8[80 \%]$ & $34[89.5 \%]$ & $9[100 \%]$ & $11[73.3 \%]$ & $62[86.1]$ \\
\hline \multirow{2}{*}{$\begin{array}{c}\text { Q6. Have you used CBCT in your work before } \\
\text { with any of your patients }\end{array}$} & Yes & $2[20 \%]$ & $4[10.5 \%]$ & $0[0 \%]$ & $4[26.67 \%]$ & $10[13.89 \%]$ \\
\cline { 2 - 7 } & No & $5[50 \%]$ & $23[60.5 \%]$ & $3[33.3 \%]$ & $9[60 \%]$ & $40[55.6 \%]$ \\
\hline
\end{tabular}


Most of the $6^{\text {th }}$ year students $58.3 \%$ [n=28], and $50 \%$ of interns [n=12] did not use CBCT with any of their patients before. Mean use in 6th year students $0.54 \pm 0.74$, one time is the higher frequency of use [31.2\%]. Mean use in dental interns was $1.16 \pm 1.43$, where 2-3 times were the higher frequency of use [16.7\%] each. Independent T-test showed significant difference between mean use of sixth years and dental interns $[\mathrm{P}=0.017]$. Comparison between males and females showed that $42.1 \%[\mathrm{n}=8]$ males and $60.4 \%[\mathrm{n}=32]$ females did not use CBCT with their patients, with mean use in males 1.26 and females 0.56 with statistically significant difference
$[\mathrm{P}=0.013]$.

Majority of participants $93.8 \%$ [n=45] sixth year students, and $83.3 \%[n=20]$ interns, obtained knowledge of CBCT in their classes with no significant difference [P=0.173]. other sources of information revealed were seminars; $22.9 \%$ [n=11] sixth year students and $50 \%[\mathrm{n}=12]$ interns, with statistically significant difference $[\mathrm{P}=0.02]$. Followed by information obtained from the internet; $35.4 \%$ [ $n=17]$ sixth year students, and $45.85 \%$ [ $n=11]$ interns. None of the participants reported any other sources of knowledge.

Table 2: Participants opinion regarding the use of $\mathrm{CBCT}$ in different fields of dentistry.

\begin{tabular}{|c|c|c|c|c|}
\hline Dental Specialties & 6th Year: $\mathbf{n}[\%]$ & Interns: $\mathbf{n}[\%]$ & Total: $\mathbf{n}$ [\%] & P Value \\
\hline All fields of dentistry & $17[35.4 \%]$ & $13[54.2 \%]$ & $30[41.7 \%]$ & 0.128 \\
\hline Prosthodontics & $16[25 \%]$ & $6[33.3 \%]$ & $22[30.6 \%]$ & 0.469 \\
\hline Endodontics & $16[33.3 \%]$ & $9[37.5 \%]$ & $25[34.7 \%]$ & 0.726 \\
\hline Maxillofacial surgery & $21[43.8 \%]$ & $7[29.2 \%]$ & $28[38.9 \%]$ & 0.231 \\
\hline Orthodontics & $7[14.6 \%]$ & 0 & $15[20.8 \%]$ & $0.014 *$ \\
\hline Will not be used & $10[20.8 \%]$ & $5[20.8 \%]$ & $3[4.3 \%]$ & 0.114 \\
\hline No idea & $3[6.2 \%]$ & 0 & & \\
\hline
\end{tabular}

Table 2 shows participants' opinion about the use of CBCT in different dental fields. Highest percent [41.7\%] was reported in all fields of dentistry, followed by maxillofacial surgery [38.9\%], and endodontics [34.7\%].
Considerable number of the respondents were willing to receive extended information and practice in CBCT imaging as shown in (Figure 5). No significant difference was reported between males and females $[\mathrm{P}=0.302]$, or between sixth year students and interns $[\mathrm{P}=0.18]$.

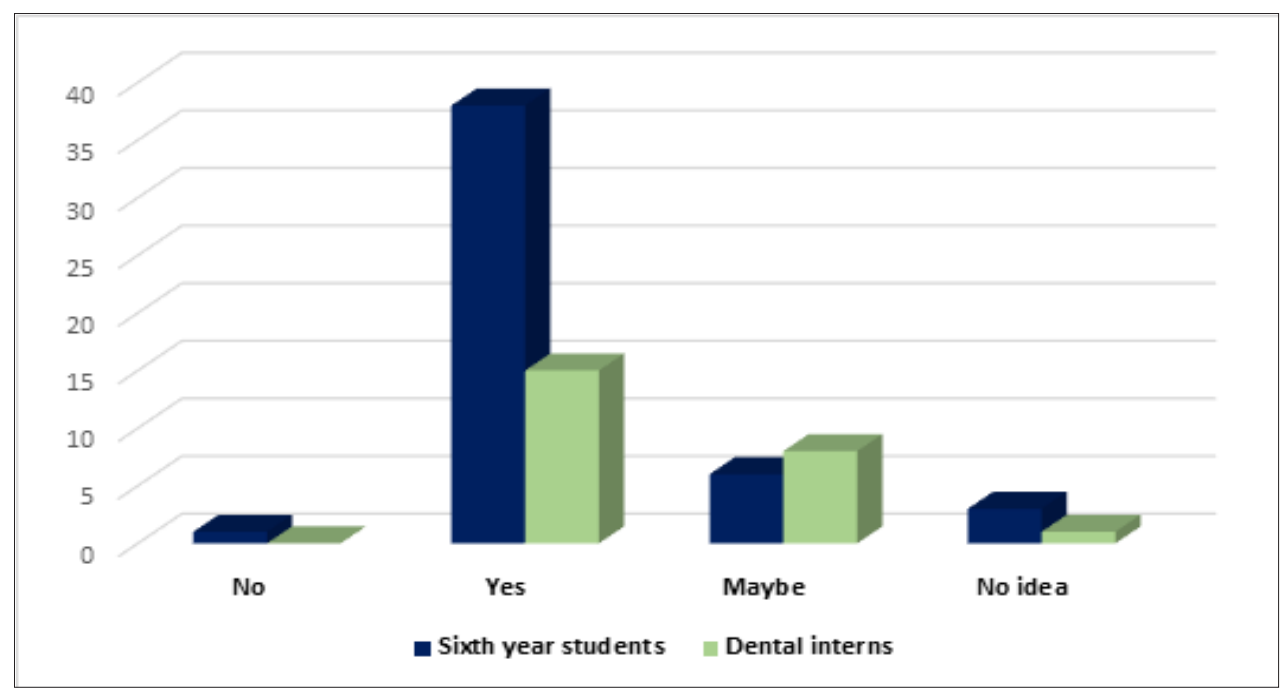

Figure 5: Bar chart students' response to question: Are you willing to receive extended information and practice in CBCT imaging.

In response to the questions of section three regarding identification of anatomical landmarks in panoramic radiography, high level of correct identifications of the marked anatomical landmarks was reported for both sixth-year students and dental interns with average correct score $91.65 \%$ and $91.7 \%$ respectively (Figure 6).
However, moderate level of correct identification of cross section views and marked anatomical landmarks in CBCT radiography for both sixth-year students and dental interns with an average correct answers $51.56 \%$ each as shown in (Figure 7). Significant differences in identification of sagittal plane $[\mathrm{P}=0.021]$ was reported. 


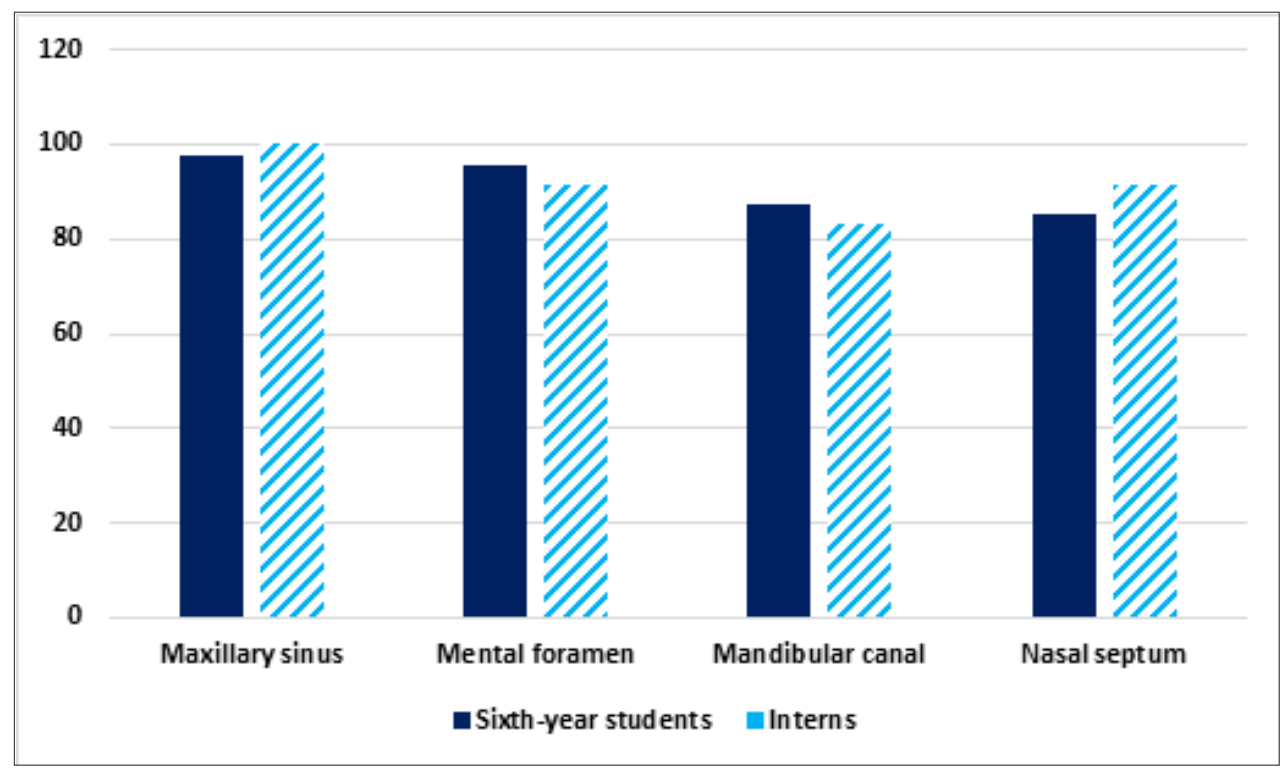

Figure 6: Bar chart percent of correct identification of anatomical landmarks in panoramic radiography of sixth year students and dental interns.

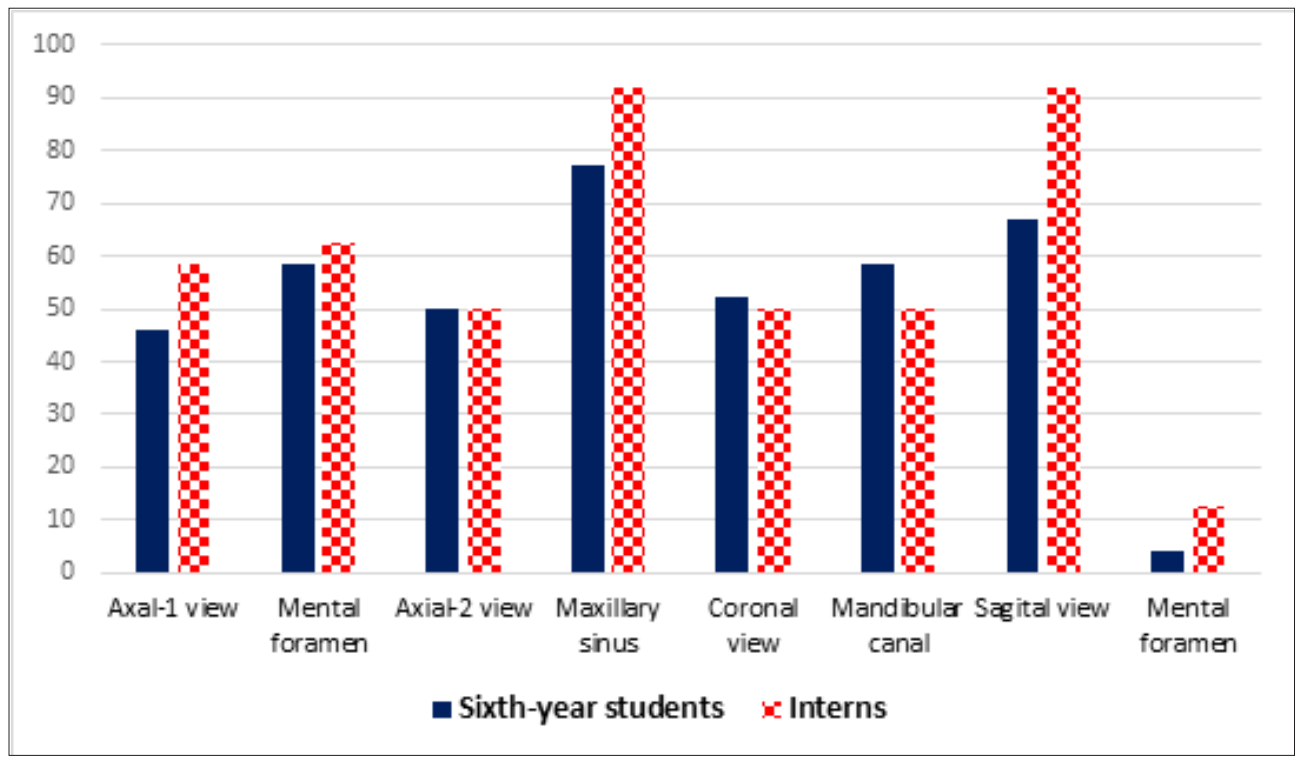

Figure 7: Bar chart percent of correct identification of cross-sectional view and marked anatomical landmarks in CBCT radiography.

A two-sample t-test of proportions showed significant difference between the proportion of correctly identified anatomical landmarks in panoramic radiography and CBCT of sixth year students [ $\mathrm{t}=9.5, \mathrm{P}=0.0005]$, and dental interns [ $\mathrm{t}=6.724, \mathrm{P}=0.0005]$. Thus, the null hypothesis was rejected and there was difference between knowledge of panoramic radiography and CBCT.

\section{Discussion}

CBCT has been considered a promising technology that is suited for use in clinical dental practice. CBCT imaging is useful in the assessment of growth and development. Its value in endodontics, implant planning, surgical assessment of pathology, TMJ assessment and pre- and postoperative assessment of craniofacial fractures has been reported. Although shortcoming may be considered when using this technology for soft- tissue imaging [7].

An understanding of the anatomical landmarks is necessary to enhance diagnostic ability. Students' knowledge regarding panoramic radiography was assessed in a number of previous reports $[8,9]$. Studies assessing students' knowledge and attitude regarding $\mathrm{CBCT}$ have focused on students' responses towards radiographic techniques, applications, and radiation doses [1013]. To the authors' knowledge, there have not been any reports analysing students' level of understanding of anatomical findings in CBCT images. The target sample chosen for this study were the 
final year dental students and freshly graduated dental interns who study in their final year curriculum an annual didactic course on dental implantology. Thus, they are expected to appreciate the importance of interpretation of anatomical landmarks for implant treatment planning.

Panoramic radiographs have been used widely as a diagnostic imaging technique; however, accurate evaluation of hard tissue morphology and density is difficult because of the variable degree of distortions, in addition to the lack of information about buccolingual cross-section dimension or the inclination of the alveolar ridge [2]. The international congress of oral implantologists 2012, supported the use of CBCT in dental implant treatment planning [14]. Accordingly, the aim of the current study was to assess and compare knowledge and attitude of sixth year dental students and interns regarding digital panoramic and CBCT radiography.

The study targeted all male and female sixth-year students and dental interns. A previous study by Al Noaman [11], assessed the knowledge and attitude of CBCT in undergraduate and post graduate female dental students only, while this study compared between responses of both male and female students.

The majority of participants in this study were females, although that a CBCT facility is available in both male and female campuses. This reflected the demography of the faculty as a whole and the direct accessibility of the authors to the participants, however no differences in most of the responses were found among female and male students in both levels of education. This high rate of female's response compared to males is in concurrence with the study carried out in Turkey on pre-graduate and post-graduate dental students in two institutions in Ankara [13].

Knowledge regarding panoramic radiography showed highest level of correct interpretation. These results reflected the familiarity of the students to panoramic radiography and agrees with their admission of the routine use of panoramic radiography with their patients. These results differ from another study carried in Japan that reported only 53\% rate of correct answers of dental students in understanding normal panoramic anatomy. This might be attributed to the difference in the data required in that study with the need of identification of bone, soft tissue and ghost images in the radiographs [8]. A different study in Queensland, Australia reported varying range of correct answers between in revealing radiographic anatomy, positioning errors, and pathology anomalies related to panoramic radiographs [9].

Most of the respondents recognise the use of CBCT in implant planning, however, many of them did not used CBCT with their own patients before. Higher percentage of the interns reported the use CBCT. A reasonable finding that reflects that the interns are close to enter their actual professional careers and are more liberated to choose their own techniques not restrained by the requirements of the college curriculum. Despite the moderate rate of utilization of CBCT by the undergraduates, still they showed higher rate of use than previous studies in which the students did not have any access to CBCT unit in their colleges $[10,13]$.
With respect to the sources of information about CBCT as reported by the participants, the majority acquired much of their knowledge in faculty classes which exposes the importance of the curriculum design at this level. The interns reported higher level of seminars attendance, being graduated renders them more flexible to attend extra faculty courses and training sessions. These results differ from Qurashl et al. [12], where minimum percent of their interns gained CBCT information from attending seminars, or through using the internet. Current results differed as well from Noaman [11], who reported that none of the postgraduates attended any seminars related to CBCT, in contrary to the undergraduates who attended CBCT-related courses. Kamburoglu et al. [13] reported that majority of the postgraduates learned about CBCT in seminars [13]. Dental students at Dow university stated that textbooks were their main source of information [10].

In the present study, sixth-year students thought that CBCT would be used most in maxillofacial surgeries and least in orthodontics. On contrary, most of dental interns supposed that CBCT would be used in all fields of dentistry, and none of them thought it would be used in orthodontics. In Mangalore, India, the interns reported that CBCT would be used in all the fields specified in the questionnaire, while least suggested its use in orthodontics [12]. The low choice for orthodontics might be attribute to the fact that undergraduates study orthodontics basics, a branch that requires further long postgraduate study to gain deep insights. The advocated use of CBCT in all fields of dentistry agrees with the studies at Dow University [10], and in Turkey [13]. Results are rather different from the previous study carried out by Noaman [11] where the higher field of CBCT application reported by the undergraduates was implant dentistry and by the postgraduates was maxillofacial surgeries and dental implants [11].

Many participants were willing to receive extended information and practice on CBCT imaging which suggested the awareness of the new technologies and their applications in different fields of dentistry and encourages extended learning programs and workshops. Dental interns in Mangalore, India [12], also suggested the need of frequent workshops to acquire more knowledge about CBCT.

In response to the last section of the questionnaire concerned with identification of the cross-section view and anatomical landmarks in CBCT radiography, a generally moderate correct answers were reported for both levels of education which indicated awareness of the participants of this type of radiology but who are in need of more practice and training for better diagnosis and treatment planning. It was noticed that the highest correct identification was of the maxillary sinus in the axial view. The lowest was the mental foramen in the sagittal view where most of the answers were "I don't know" and to a lesser extend confused with mandibular canal. The fact that the participants did not have access to the whole volume of the CBCT radiography might be one of the reasons contributing to the misjudgment, in addition to the minimum use of CBCT reported. 
These results indicate that dental curriculum designers should consider the increased CBCT practice and training in the undergraduate levels, side by side with the theoretical knowledge and to provide education focused on landmarks with lower rates for correct answers.

\section{Conclusion}

CBCT is one of the most significant developments in modern dentistry, which should be properly understood by dentists and dental students. This study was carried out in a dental institution that has a CBCT facility, yet the present study showed that final year dental students and interns were more used to panoramic radiography than CBCT. Students showed moderate awareness of CBCT implant-relative anatomy which suggests that more knowledge of CBCT should be gained through regular continuing education programmes, post graduate education courses, and meetings and seminars to update dentists' knowledge and improve their understanding of radiographic images.

\section{Appendix 1}

Part [1]: Personal information

1. l-Gender* Male [] Female. []

2. University of graduation*

3. 2- Year of graduation*

Part [2]: General information and attitude toward Panoramic and CBCT radiographs

1. What does panoramic mean?*

Narrow view [ ] Wide view. []

Focused view [] Other: []

2. Do you use Panoramic Radiograph in your routine examination of patients? *

Yes [] No [] Maybe []

3. Do you think panoramic radiographs are ideal for implant planning?

Yes [ ] No []

4. Have you heard of CBCT used specifically for Implant planning? *

Yes [] No []

5. What does CBCT abbreviation stands for? *

6. Have you used CBCT in your work before with any of your patients? *

Yes [] No []

7. If YES to previous question, how many times?

8. How do you obtain information regarding CBCT? * [Multiple responses are allowed.]

Faculty lessons []. Seminars []. Internet []

Others. [] Please specify

9. To what extent do you think CBCT will be used in routine dental practice in the near future? *

[Multiple responses are allowed.]

In all areas of dentistry []

Prosthodontics. []

Endodontics. []

Oral and maxillofacial surgery [ ] 


\section{Orthodontics []}

It will not be commonly used in routine practice []

No idea [ ]

10. Are you willing to receive extended information and practice in CBCT imaging? *

Yes [] No []

Maybe [] No idea []

Part [3]: Identifying anatomical landmarks related to implant surgery in Orthopantomogram [OPG]

You will find orthopatogram images of partially edentulous patients retrieved from the records of Oral and Maxillofacial Radiology Department. If you, please identify marked anatomical structures in each image. You will find the same image twice, the top one with a mark on the identifiable structure, the bottom one without any marks for clarification.

Identify structures marked:

(1) Case 1*:

(2) Case 2*:

(3) Case 3*:

(4) Case $4^{*}$ :

Part [4]: Identifying anatomical landmark related to implant surgery in CBCT

These are CBCT images of partially edentulous patients retrieved from the from the records of Oral and Maxillofacial Radiology Department. If you, please choose the type of view in each image and identify the marked anatomical structure.

Type of View: *

Axial []

Coronal []

Lateral. []

Panoramic []

No Idea. []

Marked Anatomical structure:*

\section{References}

1. Elani HW, Starr JR, Da Silva JD, Gallucci GO (2018) Trends in dental implant use in the U.S., 1999-2016, and projections to 2026. J Dent Res 97(13): 1424-1430.

2. Bagchi P, Nikhil J (2012) Role of radiographic evaluation in treatment planning for dental implants: A review. J Dent Allied Sci 1(1): 21-25.

3. Athota A, Gandhi Babu DB, Nagalaxmi V, Raghoji S, Waghray S, et al. (2017) A comparative study of digital radiography, panoramic radiography, and computed tomography in dental implant procedures. J Indian Acad Oral Med Radiol 29(2): 106-110.

4. Shahidi S ZB, Abolvardi M, Akhlaghian M, Paknahad M (2018) Comparison of dental panoramic radiography and cbct for measuring vertical bone height in different horizontal locations of posterior mandibular alveolar process. J Dent (Shiraz) 19(2): 83-91.

5. Bathwarr N, Nahar P (2015) Diagnostic imaging in implant dentistry: A review. J Pacif Acad High Edu Res 6(4): 32-40.

6. Ozalp O, Tezerisener HA, Kocabalkan B, Büyükkaplan UŞ, Özarslan MM, et al. (2018) Comparing the precision of panoramic radiography and cone-beam computed tomography in avoiding anatomical structures critical to dental implant surgery: A retrospective study. Imaging Sci Dent 48(4): 269-275.

7. William CS, Farman AG, Predag S (2006) Clinical applications of conebeam computed tomography in dental practice. J Can Dent Assoc 72(1): $75-80$.

8. Maeda N, Hosoki H, Yoshida M, Suito H, Honda E (2018) Dental students' levels of understanding normal panoramic anatomy. J Dent Sci 13(4): 374-377.

9. McNab S, Monsour P, Madden D, Gannaway D (2015) Knowledge of undergraduate and graduate dentists and dental therapists concerning panoramic radiographs: knowledge of panoramic radiographs. J Dent Oral Med 3(2): 46-52.

10. Iqbal W, Farooq F, Abdulbari Y, Nazir F, Quadri MA, et al. (2016) Knowledge, attitude and practice regarding computed tomography and cone beam computed tomography among dental students at dow university of health sciences. Adv Dent Oral Health 2(4): 001-0016.

11. Noaman R, Khateeb S (2017) Knowledge and attitude of cone beam CT- a questionnaire-based study among Saudi dental students. Bri J Med Med Res 19(4): 1-10. 
12. Qurashl N, Chatra L, Shenoy P, Veena KM, Prabhu R (2018) Knowledge and attitude about cone beam computed tomography [CBCT] among dental interns. J Dent Res 64: 19-25.

13. Kamburoglu K, Kursun S, Akarslan ZZ (2011) Dental students' knowledge and attitudes towards cone beam computed tomography in Turkey. Dentomaxillofac Radiol 40(7): 439-443.
14. Benavides E, Rios HF, Ganz SD, An CH, Resnik R, et al. (2012) Use of cone beam computed tomography in implant dentistry: The International Congress of Oral Implantologists consensus report. Implant Dent 21(2): 78-86. 